\title{
CDASA-CSMAVA: Contention Differentiated Adaptive Slot Allocation CSMA-CA for Heterogeneous Data in Wireless Body Area Networks
}

\author{
Fasee Ullah ${ }^{1}$, Abdul Hanan Abdullah', Gaddafi Abdul-Salaam ${ }^{3 *}$, Marina Md Arshad ${ }^{2}$ and \\ Farhan Masud $^{2}$ \\ ${ }^{1}$ Department of Computer Science and Information Technology, Sarhad University of Science and Information \\ Technology, Peshawar, Pakistan. \\ ${ }^{2}$ Faculty of Computing, Universiti Teknologi Malaysia, 81310 Skudai, Johor, Malaysia. \\ e-mail : faseekhan@gmail.com, \{hanan,marinama\}@utm.my, farhan.contact@gmail.com \\ ${ }^{3}$ Department of Computer Science, Kwame Nkrumah University of Science \& Technology, Kumasi, Ghana. \\ gaddafi.ict@knust.edu.gh ${ }^{3 *}$ \\ *Corresponding author: Gaddafi Abdul-Salaam
}

Received December 13, 2016; revised March 23, 2017; revised June 9, 2017; revised August 7, 2017; accepted September 15, 2017; published December 31, 2017

\begin{abstract}
The implementation of IEEE 802.15.6 in Wireless Body Area Network (WBAN) is contention based. Meanwhile, IEEE 802.15.4 MAC provides limited 16 channels in the Superframe structure, making it unfit for $\mathrm{N}$ heterogeneous nature of patient's data. Also, the Beacon-enabled Carrier-Sense Multiple Access/Collision-Avoidance (CSMA/CA) scheduling access scheme in WBAN, allocates Contention-free Period (CAP) channels to emergency and non-emergency Biomedical Sensors (BMSs) using contention mechanism, increasing repetition in rounds. This reduces performance of the MAC protocol causing higher data collisions and delay, low data reliability, BMSs packet retransmissions and increased energy consumption. Moreover, it has no traffic differentiation method. This paper proposes a Low-delay Traffic-Aware Medium Access Control (LTA-MAC) protocol to provide sufficient channels with a higher bandwidth, and allocates them individually to non-emergency and emergency data. Also, a Contention Differentiated Adaptive Slot Allocation CSMA-CA (CDASA-CSMA/CA) for scheduling access scheme is proposed to reduce repetition in rounds, and assists in channels allocation to BMSs. Furthermore, an On-demand (OD) slot in the LTA-MAC to resolve the patient's data drops in the CSMA/CA scheme due to exceeding of threshold values in contentions is introduced. Simulation results demonstrate advantages of the proposed schemes over the IEEE 802.15.4 MAC and CSMA/CA scheme in terms of success rate, packet delivery delay, and energy consumption.
\end{abstract}

Keywords: WBAN, IEEE 802.15.4, MAC, CSMA/CA, Patient's data, Contention 


\section{Introduction}

$\mathbf{W}_{\text {BAN has revolutionized the way in which heterogeneous vital signs of patients' data are }}$ collected. These vital signs, include temperature, blood pressure, electrocardiogram (ECG), electroencephalogram (EEG), respiratory rate, glucose level and heartbeat rate [1-2], and are monitored using BMSs. The BMS are deployed on a patient's body in one of three methods as shown in Fig. 1. Implantable BMS (e.g. endoscopy sensor) - deploying inside a patient's body for monitoring internal organs [3-4]. Wearable BMS - attaching to a shirt or skin of a patient's body for monitoring outside organs such as temperature, blood pressure, heartbeat [5], and off-Body sensors are deploying near a patient's body for monitoring sleep duration, body position, arms direction, handshaking of patients [6]. The sensed data are then transmitted to a Body Coordinator (BC), which transmits same to the medical doctors for analysis and treatments.

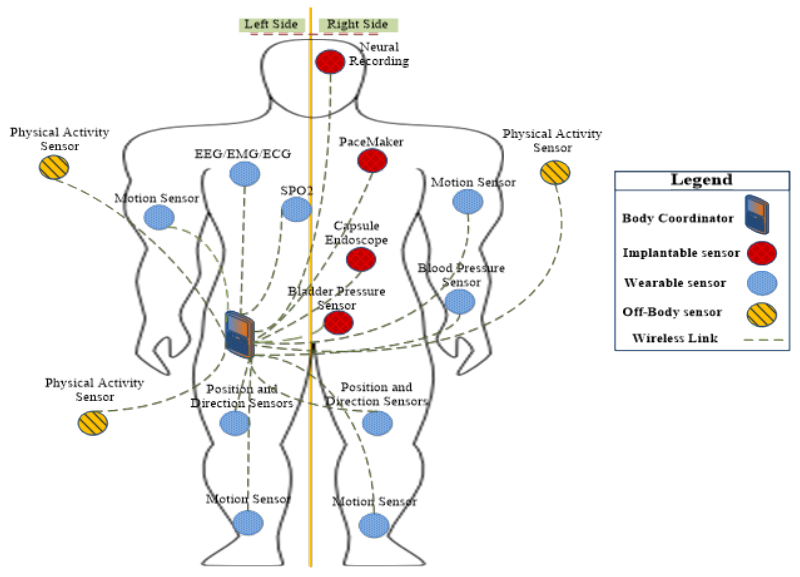

Fig. 1. Typical Example of using BMSs for a Patient

Monitored data is categorized into Non-emergency, and emergency data, [2] And results of vital signs require higher data rates for transmission, as well as reduced channel contention, overlapping, packet lost and delay. For instance, ECG (16 leads) needs 288 kbps and temperature needs 120 bps data rates [7]. The channels (slots) allocation policy to BMSs is based on using multiple scheduling access schemes, which are CSMA/CA (or Contention-based Slots allocation) and TDMA (time-division multiple access) (or Reservation-based Slots allocation) [5-6]. The TDMA is not suitable for the periodic changes in the network topology because at each time, the BMS requires synchronization with the BC in order to transmit the patient's data, which causes serious energy and delay problems. Using CSMA/CA, increases the energy consumption of BMSs due to contention-based slot allocation [7-12]. This also increases number of repetition in rounds of contentions to access channel in the Contention Access Period (CAP). This is due to limited 16 channels in IEEE 802.15.4 MAC, which is not feasible for $N$ heterogeneous nature of a patient's data.The limitations in CSMA/CA reduces performance of the MAC Superframe structure in terms of a higher data collision. In this way, BMSs re-transmit lost packets, incuring delay, lower data reliability and high energy consumption [13-15]. Furthermore, the contention is based on NB (number of backoffs), BE (Backoff Exponential) and CW (Contention Window) as reflected in Equation 1 [16]. 


$$
\mathrm{NB}=0 \text { To } 2^{\mathrm{BE}}-1
$$

where NB is the number of tries (backoffs) for accessing channel in CAP period, which depends on the values of $\mathrm{BE}$. The default minimum and maximum values of $\mathrm{BE}$ is $\operatorname{MacMinBE}=3$ and $a M a x B E=5$, respectively. With these values, the rounds of contention of each BMS increases when accessing channel in CAP period. The contention-based channel allocation to a BMS is the repetitive process where a BMS tries many times to contend and access channel in the CAP period. In each contention, the values of the previous rounds of contention are repeated in the current rounds. These increased repetitions, the collision becomes higher and BMSs re-transmit the lost packets by facing a higher delay and energy consumption, which is not feasible for emergency data. The CSMA/CA scheme drops the patient's data by exceeding the threshold values of contention. However, the dropping of a patient's data may ruin the patient's life, which is not acceptable. The CSMA/CA scheme does not allocate channels on the priority-basis to BMSs if they are contending in the final rounds. Thus, fully contention based standard IEEE 802.15.6 does not prioritize the patient data traffic, resulting in the aforementioned drawbacks in WBAN environments. Partially contention based 802.15.4 is considered for improvement in WBAN environments focusing on traffic prioritization.

In this context, this paper proposes LTA-MAC Superframe structure to provide sufficient slots and allocates dedicated slots to emergency and non-emergency based BMSs by introducing new periods to minimize the dropping of a patient's data. The paper also proposes a CDASA-CSMA/CA scheme with an enhanced equation to reduce the repetition processes and improves performance of a CSMA/CA scheme in terms of minimum collision, decrease number of packet lost, and reduces energy consumption of BMSs. The CDASA-CSMA/CA assigns priority-based channel to a BMS if it contends to access channel in the final round of contention. Then, the LTA-MAC Superframe structure accommodates the maximum number of BMSs for monitoring of the vital signs as compared to the existing standard MACs. Therefore, the main contributions of this paper are as follows:

- design of the 128 channels using operating frequency $2.4 \mathrm{GHz}$

- design of the MAC Superframe structure by introducing new periods

- Allocation of dedicated slots for emergency and non-emergency data

- Reduction of the repetition in rounds of contention of BMSs in CAP period using Equation 2.

$$
\mathrm{NB}=2^{\mathrm{BE}-1} \text { To } 2^{\mathrm{BE}}-1
$$

- Improves data packet delivery, decreases the packet loss, delay ratios and energy consumption of the body coordinator and BMSs.

The rest of this paper is organized as follows: Section 2 reviews the related works. Section 3 presents our network model and assumptions. Section 4 presents the operating frequencies, the LTA-MAC protocol and the CDASA-CSMA/CA scheme operations in details. Performance of the proposed protocol is presented in Section 5. Finally, concluding remarks are provided in Section 6.

\section{Related Work}

The MAC Superframe structure of IEEE 802.15.4 can support and allocate slots to the heterogeneous nature of patients data, and has been used by many researchers. Some recent works on MAC Superframe structure are discussed in the following. 
The suggested MAC in [17] classifies patient's data into Emergency Data (ED), Periodic Data (PD) and Normal Data (ND). In the contention for accessing channel in the CAP period, BMSs wait for random amount of time and perform backoffs. However, the CSMA/CA and TDMA scheduling schemes reduce performance of MAC protocol in terms of contention for emergency-based sensors due to long waiting period of slot allocation process, which is not an appropriate solution. The Preemptive and Non-Preemptive MAC (PNP-MAC) protocol [18] modifies the MAC Superframe structure of IEEE 802.15.4 by introducing Emergency Data Transfer slots (ETSs) and Data Transfer Slots (DTSs). The paper divides the patient's data into different classes. It has the same process for slot allocation to BMSs as mentioned in [17]. However, the main drawback of this PNP-MAC is the preemption of the non-emergency data from the allocated slots and allocates these slots on arrival of the emergency data in which non-emergency based BMSs drop the patient's data. This degrades the performance of PNP-MAC in that; it retransmits the lost packets with higher delay and higher energy consumption. The Priority-based Load Adaptive MAC (PLA-MAC) protocol [19] categories the patient data into Critical data Packet (CP), Reliability data Packet (RP), Delay data Packet (DP) and Ordinary Packet (OP). In addition, PLA-MAC uses the same dedicated DTS and ETS slots for non-emergency and emergency data, respectively, as mentioned in [18]. With these dedicated slots, all four types of a patient's data perform contention to access channel in CAP period, which greatly consumes energy of the BMSs. It also increases repetitions in rounds and reduces the performance of MAC by causing higher delay with low data reliability. In [20], the Low-delay Traffic-adaptive Medium Access Control (LTDA-MAC) proposes and it has the same procedure of slots allocation to BMSs as mentioned in [17-18]. Further, the LTDA-MAC broadcasts the notification alerts in the network for stopping contention of BMSs due to the limited channels and a higher collision of data packets, which are the limitations of this scheme as aforementioned.

This suggested Adaptive and Real-Time GTS Allocation Scheme (ART-GAS) [21] provides the service differentiation and GTS slot allocation to BMSs based on the contentions. The service differentiation is associated with data-based priority and rate-based priority, which inform the body coordinator that the generated data contains an emergency data and needs a slot allocation immediately. The winning contention of a BMS as CSMA/CA hit-miss, and called it GTS hit-miss if it does not occupy a slot of the CPF period. However, the increased contention increases collision as more BMSs contend, which reduces performance of the MAC protocol in terms of delay leading to lower data reliability, and higher energy consumption. The paper by [22] proposes the Emergency-TDMA (ETDMA), Medical Contention Access Period (MCAP), Normal-TDMA (NTDMA), CAP, and Emergency Slot (ES) periods in MAC Superframe structure. The order of the periods are changed and allocated to emergency and non-emergency data based on the contention. For example, the single BMS uses ETDMA slots in an emergency situation while MCAP slots are used in the case of more than one BMS having emergency situations. Similarly, NTDMA slots are occupied for non-emergency data. The emergency-based BMS drop the patient's data under two conditions: (1) when they perform contention to access channel in the CAP period and transmit data, but cannot access channel because of other BMSs are also contending the same channel. and (2) with failure in (1), these BMSs try an ES slot by informing the body coordinator with the assistance of an alert signal in which the body coordinator receives multiple alert signals from other BMSs and drops the packets of BMSs. Clearly, this MAC creates overheads due to changing the positions of the periods and contention, which consumes a high energy of BMSs, resulting in the dropping of a patient's data. 
The paper by [23] proposes the Priority-based adaptive Timeslots Allocation (PTA) protocol which divides the CAP's channels into three phases. In PTA, each phase slots is allocated to different types of a patient's data comprising of phase- 1 slots which is occupied to emergency data, the phase-2 slots are occupied to non-emergency data and the phase- 3 slots are reserved for audio/video data. Each phase of BMSs whether they have emergency or non-emergency must perform contention to access channel. In PTA, if the phase- 1 slots are not occupied by emergency data, then other phases of data cannot get it to use, which is a limitation of the part of PTA. Another drawback in PTA is the issues of contention as aforementioned. The suggested Fuzzy Control Medium Access (FCMA) [24] based MAC exploits acquisition, fuzzy logic control and implementation phases. In FCMA, the patient's data are divided different classes. Sensory data of a patient is collected and sent to the body coordinator by using acquisition phase. Afterwards, the decision of slot allocation to BMSs is either CAP or CFP period following the rules in the fuzzy logic control phase, which is based on data rate and priority data. However, the contention-based slot allocation to BMSs reduces the performance of MAC protocol as addressed.

Clearly, the existing MAC schemes have not been considered the contention-based channel allocation issues in their designs. Each scheme only presents the CAP period allocation as in CSMA/CA without reducing the repetition in rounds of contention. This way increases contention and degrades performance of MAC protocol, which results in higher collision; the BMS retransmits the lost/collided packets causing delay, lower data reliability and high energy consumption. Therefore, there is the need for MAC protocol that can address the limitations of the existing schemes. Our proposed methods allocate dedicated slots to the patient's data by providing sufficient time of $\mathrm{BI}$, and reduce the repetition in rounds of contention.

\section{Network Model and Assumptions}

The LTA-MAC Superframe structure targets medical applications. We assume that tiny wireless BMSs are attached to the patient's body because they monitor the heterogeneous nature of vital signs of a patient's body as described in Fig. 1. All BMSs are connected in a star topology with a BC. The batteries of BMSs are not changeable and not re-chargeable but the battery backup of $\mathrm{BC}$ is long-lasting and replaceable. The $\mathrm{BC}$ device can be a smart phone or PDA.

\section{Operating Frequencies and MAC Superframe Structure}

The operating frequency $2.4 \mathrm{GHz}$ is divided into sixteen sub-operating frequency channels, the modification of the MAC Superframe structure of IEEE 802.15.4 with reduced contention and the priority-basis channels allocation to BMSs. These contributions are discussed in the following subsections.

\subsection{The Proposed Operating Frequencies for Data Transmission}

The operating frequency $2.4 \mathrm{GHz}$ is divided into sixteen sub-frequency spectrum that are 2401 $\mathrm{MHz}, 2404 \mathrm{MHz}$, and $2407 \mathrm{MHz}$ up to $2446 \mathrm{MHz}$. Each sub-frequency spectrum provides eight channels and the bandwidth of each channel is $9.375 \mathrm{MHz}$. With this type of classification, we design 128 channels with a higher bandwidth which transmit a bigger amount of data with a minimum time period. The channel interference/overlapping is avoided between two channels with the support of a guardband and its gap between the channels is 0.1 
MHz. This guardband between channels assists not to collide data [25]. The guardband between main channels is $2.0 \mathrm{GHz}$ with the same benefits as highlighted for sub-frequency channels.

\subsection{The Proposed LTA-MAC Superframe Structure}

The LTA-MAC Superframe structure provides 128 channels and comprises of a beacon (B), CAP, N (Notification), OD (On-Demand), NETS (Non-Emergency Transfer Slot), CETS (Critical-Emergency Transfer Slot), and Inactive Period (IP) / Low Power Listening (LPL) periods as shown in Fig. 2. The CAP period is allocated 25 channels, the NETS period is allocated 50 channels, and CETS period is allocated 49 channels. The channels of the proposed Superframe structure are dynamic in length and assign more channels to BMSs depending on the traffic loads. Further, the IP is used for saving energy when there is no data transmission being performed. We classify the patient's data into ordinary data packets (ODPs) and critical data packets (CDPs). OPD packets contain a normal reading that is temperature, glucose and can be delayed. CPD packets contain the life-threatening low threshold $\left(\mathrm{CDP}_{\mathrm{LTH}}\right)$ and high threshold $\left(\mathrm{CDP}_{\mathrm{HTH}}\right)$ values of vital signs which need to be delivered with minimum delay and without loss with higher reliability.

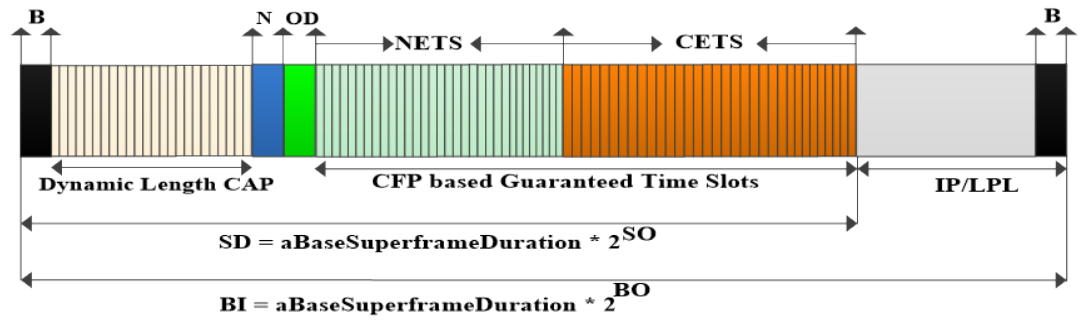

Fig. 2. The Proposed LTA-MAC Superframe Structure

\subsubsection{Operations of the LTA-MAC Superframe Structure}

This section describes each period of the proposed LTA-MAC Superframe structure in detailed as follows:

\section{a. Beacon Frame}

The body coordinator broadcasts a beacon frame to the whole network of BMSs for establishing communication channels which contains information of the synchronization, the address of the body coordinator, the allocated time interval to the active parts of the Superframe structure and an announcement of the next beacon interval. Similarly, the exchange of the contention, notification, and on-demand beacons are transmitted between BMSs and the body coordinator under the following conditions.

i. The BMSs broadcast a beacon frame during contention to access channel in the CAP period. In contention, each BMS scans different available channels in the CAP period. On the successful allocation of the CAP's channel, the body coordinator replies back with an acknowledgment to that particular BMS for transmitting data in NETS/CETS slots of the CFP period.

ii. The body coordinator transmits the notification (N) frame to those BMSs that have already been allocated NETS/CETS slots when there is no empty slot available. With this notification frame, the BMSs de-allocate the occupied slots. 
iii. The BMs drops the patient's packets if it does not access channel in the CAP period in IEEE 802.15.4 MAC Superframe structure. To avoid dropping data packets in the LTA-MAC Superframe structure, the particular BMS uses OD beacon for transmitting data to NETS/CETS slots, accordingly.

iv. The second benefit of the OD beacon is that the medical doctor can retrieve the sensory reading of any BMS with the support of the body coordinator.

\section{b. Header of the Beacon frame}

The header structure of the beacon frame includes type, synchronization preamble sequence (SHR-PS), synchronization start of frame Delimiter (SHR-SD), MAC header beacon sequence number (MHR-BSN), destination address, source address, CAP channels and frame check sequence (FCS) as shown in Fig. 3. The type field occupies 3 bits and its value is 000 or $(C M 7 / B C N)$ which indicates the beacon frame is broadcasted from the body coordinator to BMSs. The SHR-PS occupies 8 bits and is a signal for clock synchronization between BMS and the body coordinator. The body coordinator uses $S H R-S D$ for showing to the receiver BMSs that is the start of a beacon frame. The SHR-SD field occupies 8 bits in the beacon frame. During the association steps of a BMS with the body coordinator, the BMS transmits more than one association frames, which is maintained and updated with the support of the $M H R-B S N$. The body coordinator reserves 4 bits for MHR-BSN field. Similarly, the body coordinator assigns 8 bits to the destination and source addresses, respectively. In this case, the source address is the BMS while the destination address is the body coordinator. During broadcasting of a beacon frame to the network, the body coordinator uses FFFFFFFF as destination addresses. The CAP field is used for contention. The FCS occupies 16 bits and carries the frame in a sequence to the destination.

\begin{tabular}{|c|c|c|c|c|c|c|c|c|}
\hline $\begin{array}{c}\text { Beacon } \\
\text { Frame } \\
\text { Header }\end{array}$ & Type & $\begin{array}{c}\text { Synch. Preamble } \\
\text { Sequence }\end{array}$ & $\begin{array}{c}\text { Synch. Start of } \\
\text { Frame Delimiter }\end{array}$ & MHR BSN & $\begin{array}{c}\text { Destination } \\
\text { Address }\end{array}$ & $\begin{array}{c}\text { Source } \\
\text { Address }\end{array}$ & CAP & FCS \\
\hline
\end{tabular}

Fig. 3. Header of the Beacon frame

\section{c. Header of the Notification frame}

The header of the notification frame comprises of six fields that is type, notification sequence (NS), destination address, source address, Superframe specification (SFS) and FCS as depicted in Fig. 4. Each field occupies different bits as indicated. The body coordinator broadcasts or sends the type frame to those BMSs that have occupied the guaranteed timeslots (GTSs) of the CFP period. The value of the type frame is 100 which indicates the notification frame and has come from the body coordinator regarding the de-allocation of NETS or CETS slots. The destination address shows the address of the body coordinator while the source address can be a specific address or it can be broadcast address. The FCS verifies the integrity of the transmitted frame.

\begin{tabular}{|c|c|c|c|c|c|}
\hline Notification & 3 & 8 & 8 & 3 & 16 \\
\hline Frame & Type & $\begin{array}{c}\text { Destination } \\
\text { Address }\end{array}$ & $\begin{array}{l}\text { Source } \\
\text { Address }\end{array}$ & $\begin{array}{c}\text { SF } \\
\text { specification }\end{array}$ & FCS \\
\hline
\end{tabular}

Fig. 4. Header of the Notification frame 


\section{d. Header of On-demand frame}

The body coordinator can retrieve the sensory data from any BMS during monitoring of vital signs of a patient's body. With this advantage, the On-demand frame header comprises of six fields that are type, destination address, source address, security header, payload and FCS as depicted in Fig. 5. Each field of the header occupies different bits. The first field of the header is the type and its assigned value is 101 . When a body coordinator transmits on-demand frame to any BMS then that particular BMS searches for 101 value in the type field which indicates for requesting of sensory data. The destination address indicates the address of the particular BMS and the source address is the address of the body coordinator. The data payload is occupied for sensory data. The maximum length of the data payload is 15360 bits. The function of FCS field is the verification of the integrity of the received frame on the side of the body coordinator.

\begin{tabular}{|c|c|c|c|c|c|}
\hline On-Demand & 3 & 8 & 8 & M & 16 \\
\hline Frame & Туре & $\begin{array}{c}\text { Destination } \\
\text { Address }\end{array}$ & $\begin{array}{c}\text { Source } \\
\text { Address }\end{array}$ & Payload & FCS \\
\hline
\end{tabular}

Fig. 5. Header of the On-demand frame

\section{e. Headers of NETS and CETS Frames}

The functionalities of header frames NETS and CETS are to allocate channels to non-emergency and emergency data, respectively. The NETS data frame header is employed for non-emergency data that is ODP packet. While CETS header frame is employed for life-threatening emergency data packets that are $C D P_{\text {LTH }}$ and $C D P_{\text {нтH. The allocation of }}$ channels to both types of sensory data is based on the contention (backoffs). Each BMS tries maximum five times backoff to access channel in CAP period. If the BMS does not get a channel access in CAP period, then that particular BMS performs twice clear channel assessment (CCA) for getting a collision-free access of the OD slot. With this supported OD slot, the BMS transmits a frame to NETS/CETS slots without dropping of a patient's data. This feature has been added in the beacon-enabled CDASA-CSMA/CA scheme which is explained later. Moreover, the header format of OD is comprised of type, start of delimiter (SFD), data sequence number (DSN), destination address, source address, data payload and FCS, as shown in Fig. 6. On the successful winning of the CAP channel and sending of sensory data, the type field indicates to a BMS for confirmed allocation of NETS/CETS slots. For this purpose, the value of type field is set 0001 which assists the body coordinator for confirmed allocation of a slot. The SFD indicates the start of the frame while DSN shows the frame sequence number if a single BMS transmits more than one frame at a time. The destination address is the address of the body coordinator and the source address is the address of the sender BMS. The data payload contains the sensory data of a patient's body, which is detected in monitoring of vital signs. The length of the data payload is 15360 bits. The last field is FCS which is used to verify the correctness of the transmitted data frame on the receiver side.

\begin{tabular}{|c|c|c|c|c|c|c|c|}
\hline \multirow{2}{*}{$\begin{array}{l}\text { NDTS } \\
\text { Data Frame }\end{array}$} & 4 & 8 & 8 & 8 & 8 & $\mathrm{Q}$ & 16 \\
\hline & Type & SFD & DSN & $\begin{array}{c}\text { Destination } \\
\text { Address }\end{array}$ & $\begin{array}{c}\text { Source } \\
\text { Address }\end{array}$ & $\begin{array}{c}\text { Data } \\
\text { Payload }\end{array}$ & FCS \\
\hline
\end{tabular}

Fig. 6. Header of the NETS and CETS Frame 


\subsection{Working of the Proposed CDASA-CSMA/CA Scheduling Access Algorithm}

The CDASA-CSMA/CA scheme is employed to minimize the repetition in rounds of contention for accessing channel and assigns channels to BMSs on the rounds priority-basis. With minimization of the repetition in rounds of contention, the collision of BMSs is reduced and enhances the performance of the CDASA-CSMA/CA scheme in terms of minimum collision, decreases the re-transmission of the lost packets, reduces delay with higher reliability, and BMSs consume minimum energy. The highlighted boxes as shown in Fig. 7 represents our contributions in the design of the CDASA-CSMA/CA scheme.

The $C W, N B$ and $B E$ are the significant parameters which may increase or decrease the rounds of contention of BMSs for accessing channel in the CAP period. We set the values of $B E$ start from 1 to 5 . First round of the contention is represented by MacMinBE1 = 1. For the first round of contention, Equation (1) of the CSMA/CA scheme is used in the implementation. In this round, all BMSs perform contention for accessing channel in ranges between 0 and 1 . We assume that a BMS has not occupied channel in the first round of contention and its value of the contention $(N B<a M a x B E)$ is true. Moreover, this paper classifies the remaining rounds of contention into four rounds, which are denoted by MacBE = 2 (second round), MacMinBE = 3 (third round), MacMedBE = 4 (fourth round) and $a M a x B E=5$ (fifth round) as depicted in Fig. 7. These rounds are implemented by using Equation (2), which reduces the overhead problems of the CSMA/CA scheme as aforementioned. For the second round of contention, the value of $N B$ is incremented by one and sets the value of $M a c B E=2$. In this case, the contention is reduced by using Equation (2) and transfers the control for obtaining channel access towards step 4 as described in lines 35 to 39 of the proposed algorithm 1 for CDASA-CSMA/CA. In step 4, the BMS performs CCA as similarly done by other BMSs in the normal execution for accessing channel in CAP period. In CSMA/CA, each BMS contends for accessing channel in ranges 0 to 3 times while the CDASA-CSMA/CA based BMS competes in ranges 2 to 3 times. Then, the body coordinator allocates NETS/CETS slots to non-emergency and emergency data on obtaining successfully the CAP channels. Furthermore, for the third round of contention, the value of $N B$ is incremented by one and sets the value of MacMinBE $=3$. In this round of contention, BMS uses the same Equation (2) and transfers the control for obtaining a channel access towards step 4. During contention for accessing channel in the third round, the repetition in rounds of contention is reduced and BMSs contend for accessing channels in ranges between 4 to7. While CSMA/CA-based BMS performs contention in ranges between 0 to 7 . Lines 41 to 45 are described in the algorithm 1 for third round.

Similarly, it is assumed for the fourth round of contention that the BMS has not obtained a channel access and the value of $N B$ is incremented by one which becomes MacMedBE $=4$. In this fourth round, the CDASA-CSMA/CA-based BMS competes for accessing channels in ranges between 8 to 15 times and reduces the rounds of contention. The standard CSMA/CA-based BMS contends for accessing channel in ranges between 0 to15, which are the repetition in rounds of contention. These repetitive rounds create overheads in terms of a higher number of collision, increases delay with high re-transmission of the lost packets and higher energy consumption of BMSs. Thus, the CDASA-CSMA/CA-based BMS transfers the control directly towards step 7 and allocates the CAP's channel on the priority-basis as described in the lines 47 to 51 of algorithm 1 of the CDASA-CSMA/CA. 


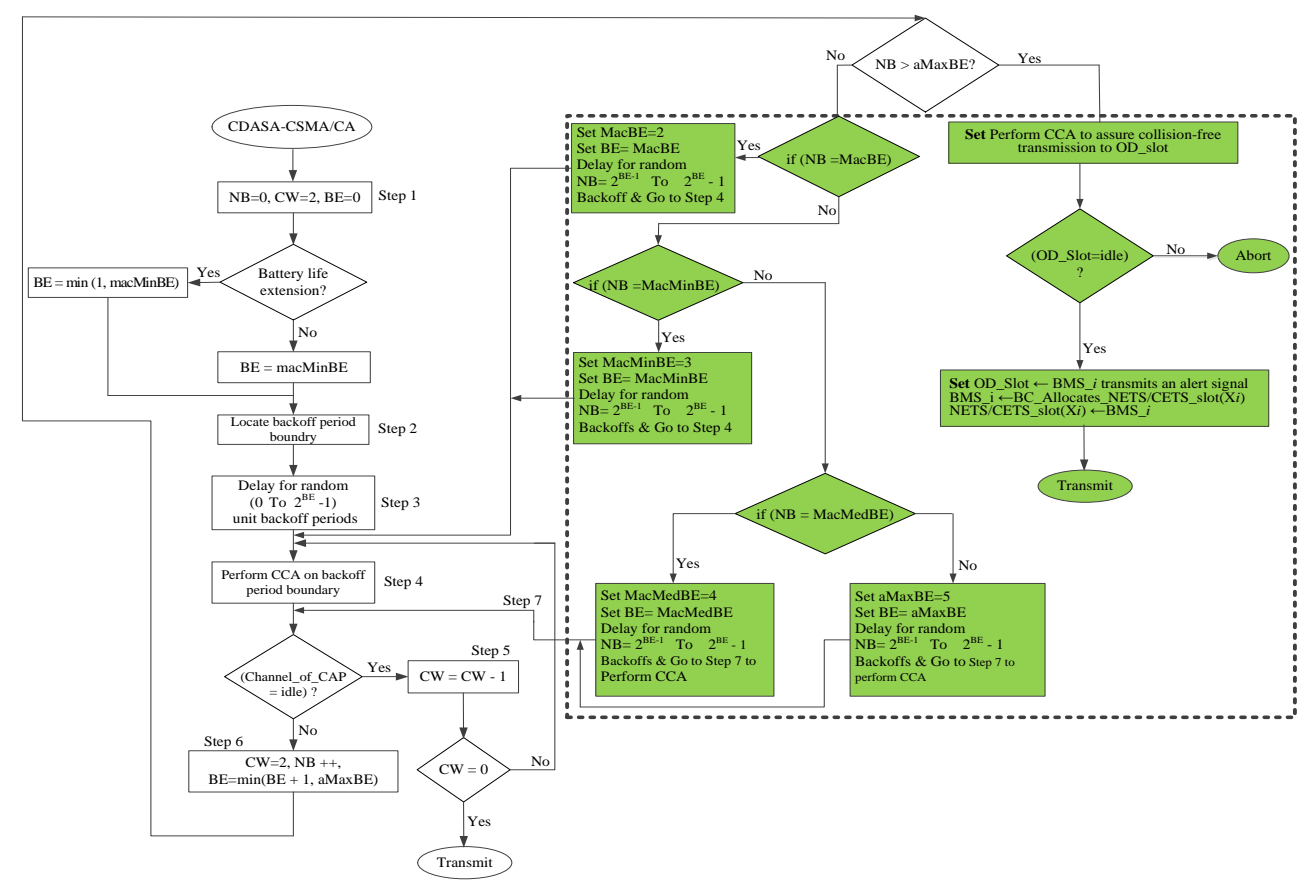

Fig. 7. The proposed Beacon-Enabled CDASA-CSMA/CA scheduling Access scheme

The fifth round for accessing channel is the final round of contention. The value of $N B$ is incremented by one and sets the value of $a M a x B E$ is 5 . Equation (2) is used in this fifth round which assists to BMSs in reducing the repetition in rounds of contention. The reduced rounds of contention are in ranges between 16 to 31. In this round, the CDASA-CSMA/CA reduces the channel assignment control steps as compared to second and third rounds, and transfers the control for accessing channel on the priority-basis towards step 7 as described in lines 53 to 56 of the algorithm 1. The CSMA/CA-based BMSs perform contention in ranges between 0 to 31 times using Equation 1. Based on these reduced rounds of contention, the proposed scheme performs better than CSMA/CA scheme. Another advanced functionality of the CDASA-CSMA/CA scheme is that it does not drop the patient's data when the value of $N B>$ $a M a x B E$ is true. This is because it gives a chance to BMS by using OD slot of the LTA-MAC Superframe structure to transmit data. With this condition, the BMS performs twice a CCA for assuring of collision-free access. On other hand, BMS must wait for the next announcement of beacon interval (BI). However, the CSMA/CA-based BMSs drop the patient's data by exceeding threshold values of contention (NB>aMaxBE). Thus, the CDASA-CSMA/CA scheme has minimized the repetition in rounds of contention for accessing channel in CAP period. These improvements in rounds of contention and the LTA-MAC Superframe structure are better performed by minimizing collision, reduces re-transmission of the lost packets, and energy consumption of BMSs. The proposed CDASA-CSMA/CA algorithm 1 is presented in the following as follows: 


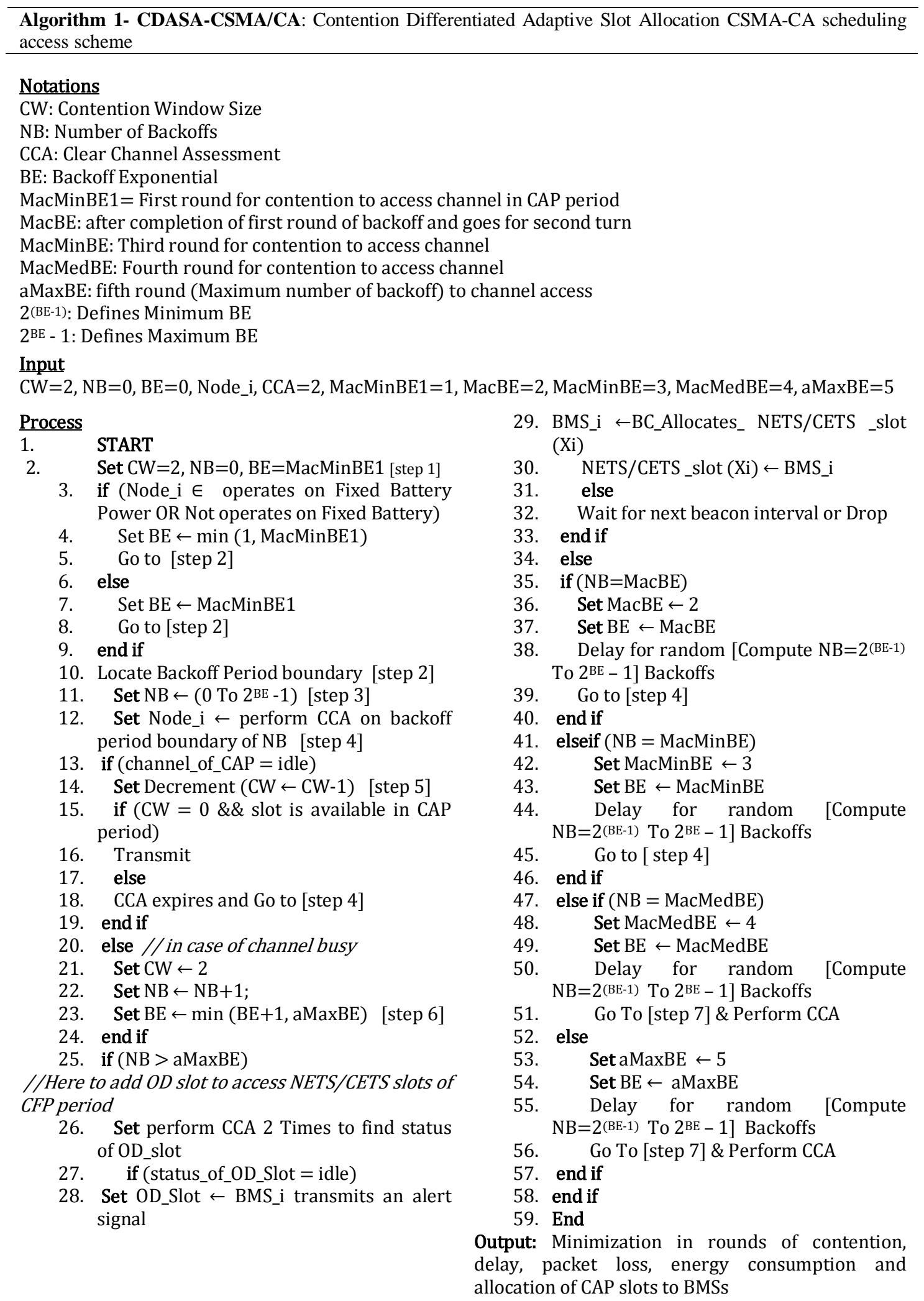




\section{Performance Evaluation}

In this section, we compare the performance of the proposed methods with the standard MAC Superframe structure and a CSMA/CA scheme in terms of on-time packets success ratio, packet loss ratio, packet delivery ratio, packet delay and energy consumption using NS-2 [26] under the package ns-allinone-2.34. Table 1 shows the parameters list used in the simulation.

Table 1. Simulation Parameters

\begin{tabular}{|l|l|l|l|}
\hline Parameter & Value & Parameter & Value \\
\hline Operating Carrier Frequency & $2.4 \mathrm{GHz}$ & Topology & star \\
\hline Channel Rate & $250 \mathrm{kbps}$ & Sending Data Rates & $20 \mathrm{kbps}$ \\
\hline No of channels in proposed MAC & 128 & MAC Payload size & 1920 bytes \\
\hline No of channels in IEEE 802.15.4 MAC & 16 & Buffer size of the Body Coordinator & 2000 bytes \\
\hline BO set for both MAC protocols & 9 & Buffer size of a BMS & 1920 bytes \\
\hline SO set for both MAC protocols & 8 & BI in seconds & 196.608 seconds \\
\hline A Slot Duration & 0.768 seconds & No. of the Body Coordinator & 1 \\
\hline Time of SF Duration (SD) & 98.304 seconds & Number of BMSs & 14 \\
\hline Low data generation rate & $0.5 \mathrm{~ms}$ & Power Consumed in Sleep & $0.005 \mathrm{~mW}$ \\
\hline Traffic Type & CBR & Power Consumed in Receive & $1.8 \mathrm{~mW}$ \\
\hline $\begin{array}{l}\text { Power Consumed in Transmission state } \\
\text { Duration of Turn-On radio to }\end{array}$ & $27-220 \mathrm{~mW}$ & $\begin{array}{l}\text { Power required for radio into receiver } \\
\text { from transmission }\end{array}$ & $0.4 \mathrm{~ms}$ \\
\hline \begin{tabular}{l} 
Transmit/Receive \\
\hline
\end{tabular} & $0.8 \mathrm{~ms}$ & Simulation Run Time & $2000 \mathrm{~seconds}$ \\
\hline
\end{tabular}

\subsection{Results and Discussion}

In this section, we present analysis and discussion of the results in the light of the mentioned parameters.

\subsubsection{On-Time Packet Success Ratio}

Fig. 8 shows the comparative performance of the average packet success rate of the CDASA-CSMA/CA scheme (green lines) with the standard CSMA/CA scheme (red lines). The CDASA-CSMA/CA reduces the repetition in rounds of contention for accessing channel in the CAP and CFP periods. For the first round of contention, the CDASA-CSMA/CA uses the same Equation (1) as used in the CSMA/CA in providing sufficient channels in the CAP period of the LTA-MAC Superframe. The collision increases in the CSMA/CA and the packet success rate becomes down when the traffic load exceeds from BMS 6. This occurs because of the limited number of channels provided in the MAC Superframe structure of IEEE 802.15.4 and BMSs exceed the threshold value of the contention ( $a M a x B E$ ). These limitations, the CSMA/CA-based BMSs are not capable to transmit packets successfully on time. Moreover, the CDASA-CSMA/CA improves the contention of the first round by achieving $12 \%$ as compared to a CSMA/CA. As the rounds of contentions increase, the packet success rate of the CDASA-CSMA/CA increases due to use of Equation (2). The second and third rounds of the contentions, the CDASA-CSMA/CA reduces the repetition values in rounds of contention for accessing the CAP's channels as compared to CSMA/CA because CSMA/CA repeats contention values of each round in the next round of contention which reduces the performance of MAC as aforementioned. Thus, the second and third rounds of the contention of the CDASA-CSMA/CA have been improved by achieving $20.94 \%$ and $27.63 \%$, respectively as compared to a CSMA/CA. As more BMSs go for contentions in the fourth and fifth rounds, the CDASA-CSMA/CA reduces the repetition in rounds for contending to access channel with the support of the LTA-MAC Superframe structure due to sufficient time of BI 
(beacon interval) and SD for allocating of slots to BMSs without collision. The collision is exceeded in the CSMA/CA when the traffic loads exceed from BMS 6 because repetition in rounds of contentions are increased for accessing channel. The CDASA-CSMA/CA achieves the improvements in the fourth and fifth rounds of contentions are $42.10 \%$ and $60.27 \%$, respectively.

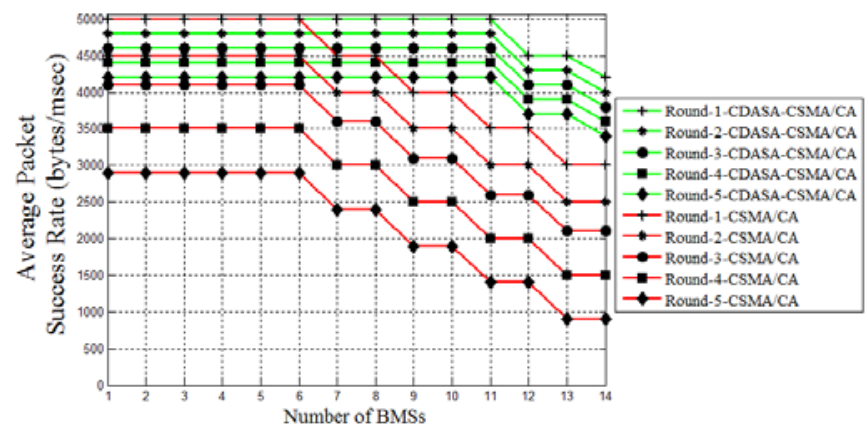

Fig. 8. Comparison of average Packet Success Ratio versus Number of BMSs between CDASA-CSMA/CA and CSAMA/CA

\subsubsection{Average Packet Loss Ratio}

Fig. 9 shows results of the average packet loss ratio of the CDASA-CSMA/CA and CSMA/CA, respectively. Both schemes generate data at a rate of 0.5 milliseconds and we compare each round of contention for packet loss. In the first round of contention, the results of the packet loss ratio of the CDASA-CSMA/CA and CSMA/CA are the same because the reduced number of rounds for accessing channel is used in CAP period. For the second round of contention, the repetition in rounds of the contention is reduced by using Equation (2) in the CDASA-CSMA/CA which assists in reducing the packet loss up to $15 \%$ as compared to a CSMA/CA. As the number of rounds increase in accessing channels in the CAP period using CSMA/CA, the packet loss ratio increases due to the limited number of channels in IEEE 802.15.4 MAC whereas BMSs cannot transmit data in the same BI. This happens due to exceeding of threshold value ( $a M a x B E)$ of contention in CSMA/CA. The third, fourth, and fifth rounds of the contention in CDASA-CSMA/CA-based BMSs have reduced by using Equation (2) for accessing channels in the CAP period. These reductions in contention, the average packet loss ratio of the CDASA-CSMA/CA performs better by reducing 33\% in the third round, $37 \%$ in the fourth round and $45 \%$ in the fifth round. In addition, the LTA-MAC Superframe structure assigns sufficient channels with a high bandwidth for accessing CAP period and transmits sensory data about a patient in the same BI of the CFP's period. Another feature is the OD_channel which assists in not dropping the patient's data after exceeding the threshold value of the contention. However, this feature is not available in the MAC Superframe structure of IEEE 802.15.4. 


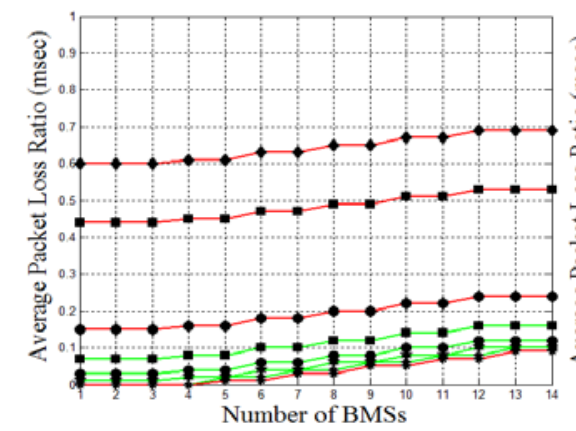

Fig. 9. Comparison of average packet loss ratio versus Number of BMSs between CDASA-CSMA/CA and CSMA/CA with low generation data rate

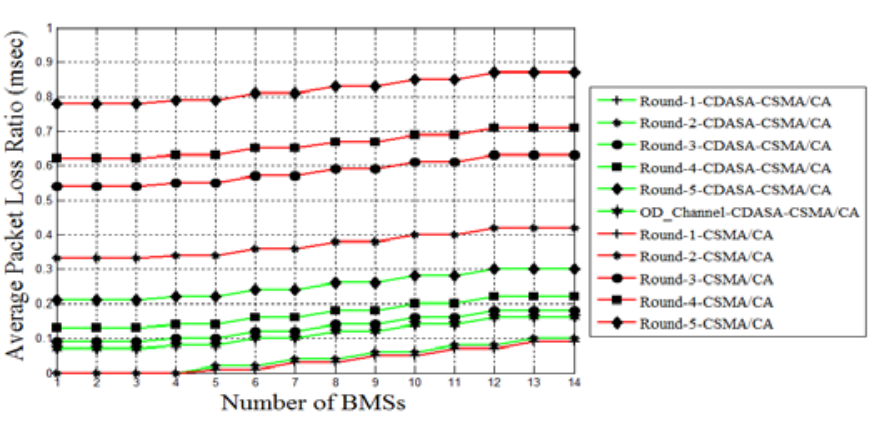

Fig. 10. Comparison of average packet loss ratio versus Number of BMSs between CDASA-CSMA/CA and CSMA/CA with high generation data rate

Fig. 10 shows the average packet loss ratio with high generation data rate of 1.5 milliseconds. BMSs generate data at high speed of 1.5 milliseconds and contend for accessing channel in the CAP period. The body coordinator assigns NETS or CETS slots of the CFP period to the BMSs that accessed a channel in the CAP period. The high speed of data generation and the average packet loss ratio of the CDASA-CSMA/CA is a bit high as compared to Fig. 9 but as compared to the CSMA/CA scheme of Fig. 10 is very minimum. For the first round of contention, both schemes have the same output and the packet loss ratio increases gradually as the traffic increases. For the second and third rounds of contentions, the CDASA-CSMA/CA performs better by reducing the repetition in rounds of contention which reduces the packet loss ratio. This is because of the provided sufficient channels and BI in the LTA-MAC Superframe structure which accepts a high generation rate of traffic for transmission. However, the repetition of values for the second round of contention are repeated in the third rounds of contentions in a CSMA/CA which reduces the performance in terms of higher collision, increases delay with high energy consumption of BMSs. Thus, the high speed of data generation, the limited number of channels and BMSs exceed the contention threshold value are the reasons for the higher number of packet loss in a CSMA/CA. The packet loss ratio is reduced in the second round by $33 \%$, the third round is by $45 \%$, the fourth round is by $49 \%$ and the fifth round is by $57 \%$ in the CDASA-CSMA/CA. In addition, the OD_channel does not drop the patient's data and assigns the CFP period when a BMS exceeds the threshold value of the contention in the CDASA-CSMA/CA.

\subsubsection{Average Packet delivery Ratio}

The average packet delivery ratio (PDR) of CDASA-CSMA/CA (green lines) is compared with a CSMA/CA (red lines), as shown in Fig. 11. Both schemes generate data at 0.5 milliseconds. The LTA-MAC Superframe structure provides 128 channels and the provided bandwidth to each channel for data transmission is $9.375 \mathrm{MHz}$. The guardband between channels is $0.1 \mathrm{MHz}$, which protects channels from overlapping. Equation (2) is used in implementation of CDASA-CSMA/CA for reducing the repetition in rounds of contention. The sufficient channels, the guardband between channels, and the reduced rounds of the contention are the advantages of transmitting a huge amount of a patient's data without interrupting each other. For the first round of contention, the average PDR of a 
CDASA-CSMA/CA achieves by $15 \%$ as compared to a CSMA/CA because MAC Superframe structure of IEEE 802.15.4 contains limited channels and CSMA/CA increases the repetition in rounds of contention as depicted in Fig. 11. For the second and third rounds of contention, the average PDR of the CDASA-CSMA/CA is achieved by $20 \%$ and 33\%, respectively as shown in Fig. 11. For the fourth and fifth rounds of the contentions, the CDASA-CSMA/CA has reduced the steps for accessing channels and the BMSs transmit data directly to step 7 by performing a CCA activity. Thus, the achieved PDR in the fourth and fifth rounds are $20 \%$ and $15 \%$ in the CDASA-CSMA/CA, respectively, as compared to a CSMA/CA.

The CDASA-CSMA/CA and CSMA/CA generate data at the rate of 1.5 milliseconds. In the first round of contention, the CDASA-CSMA/CA performs better for an efficient packet delivery and achieves improvement of $29 \%$ as shown in Fig. 12 (green lines) as compared to a CSMA/CA (red lines), which reduces delivery of the packets when the traffic loads exceed. This occurs because of the limited resources of MAC Superframe structure as aforementioned. For the second and third rounds of contentions, the average PDR of the CDASA-CSMA/CA scheme is achieved by $13 \%$ and $26 \%$, respectively, as compared to a CSMA/CA in which CSMA/CA-based BMSs repeat values of contention in the next round of contention. Moreover, the average PDR is reduced a bit in the CDASA-CSMA/CA due to high generation rate of data. But as compared the CDASA-CSMA/CA with a CSMA/CA, the CDASA-CSMA/CA performs better during the packet delivery to the body coordinator and achieves improvement in the fourth round by $28 \%$ and in the fifth round is by $19 \%$. As more BMSs exceed threshold value in the CSMA/CA, the PDR goes down due to the limited number of channels in IEEE 802.15.4 MAC and BMSs wait to transmit the patient's data in the next announcement of BI.

\subsubsection{Average Packet Delay Ratio}

The average packet delay of the CDASA-CSMA/CA is compared with the CSMA/CA as shown in Fig. 13. The data generation rate is 0.5 millisecond considered in both schemes. The dedicated slots are reserved for emergency and non-emergency based BMSs in the LTA-MAC Superframe structure which assist to reduce collision. In the first round of contention, the average packet delay is reduced by $97 \%$ (green color) using the CDASA-CSMA/CA as shown in Fig. 13. This improved performance is achieved due to the sufficient channels, the sufficient

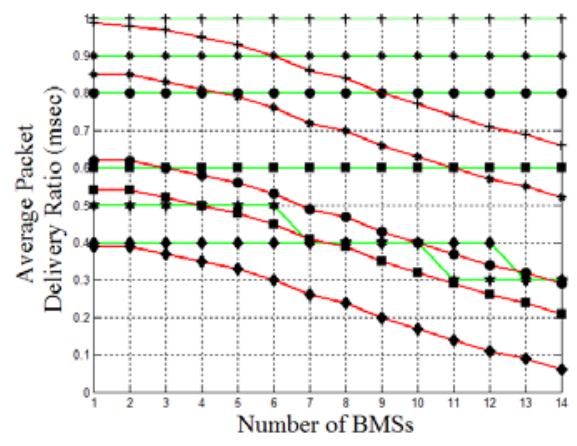

Fig. 11. Comparison of average packet delivery ratio versus Number of BMSs between CDASA-CSMA/CA and CSMA/CA with low generation data rate

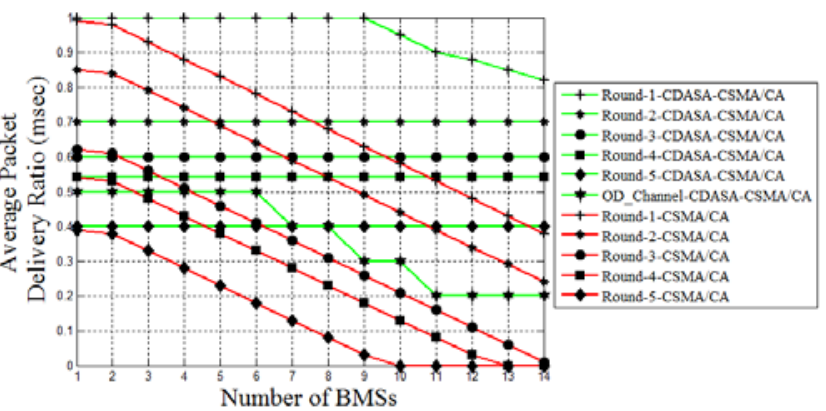

Fig. 12. Comparison of average packet delivery ratio versus Number of BMSs between CDASA-CSMA/CA and CSMA/CA with high generation data rate 
time of BI, Superframe duration (SD), and the slot duration as compared to a CSMA/CA in the first round. For second, third, fourth, and fifth round of the contention, the Equation (2) assists to reduce the repetition in rounds of the contention. Thus, the achieved reduced delay in the packet delivery is $93 \%$ in the second round, $89 \%$ in the third round, and $86 \%$ in fourth and fifth rounds. The CSMA/CA-based BMSs exceed threshold values of contention very quickly as the traffic loads increase. This degraded performance of the CSMA/CA increases the waiting time of BMSs for new BI which increases delay of the packets and the BMSs drop the patient's data.

Fig. 14 compares the average packet delay of both schemes with high generation rate of data at 1.5 milliseconds. As shown in Fig. 14, the green color represents the CDASA-CSMA/CA and the red color represents a CSMA/CA. Equation (2) has reduced repetition in rounds of the contention in the CDASA-CSMA/CA. Thus, the achievement of the average packet delay in a CDASA-CSMA/CA is reduced by $93 \%$ in the first round, $89 \%$ in the second round, $83 \%$ in the third round, $79 \%$ in the fourth round and $76 \%$ in the fifth round. However, the CSMA/CA repeats the values of rounds in each contention and BMSs do not get a channel in CAP period due to limited channels. In this way, the delay of the packet delivery is reached at a high point after exceeding the traffic loads from BMS 7, as shown in Fig. 14.

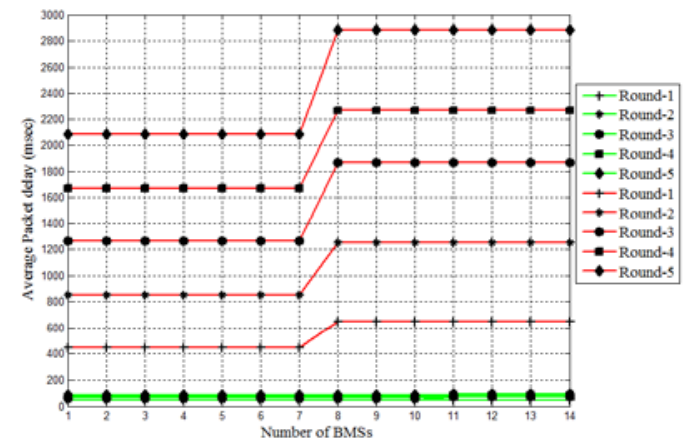

Fig. 13. Comparison of average packet delay ratio versus Number of BMSs between CDASA-CSMA/CA and CSMA/CA with low generation data rate

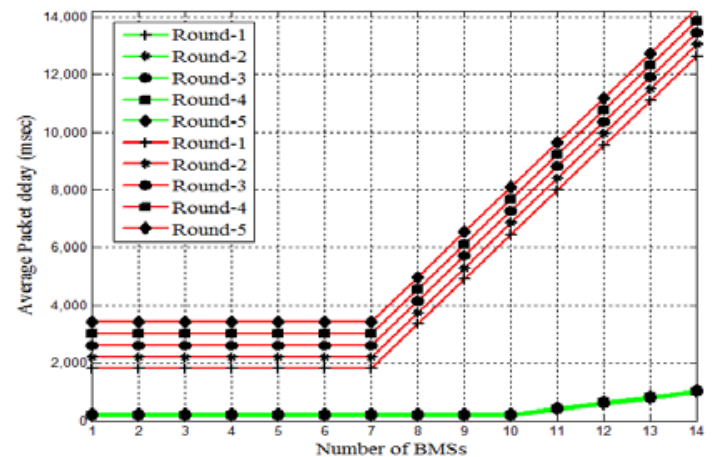

Fig. 14. Comparison of average packet delay ratio versus Number of BMSs between CDASA-CSMA/CA and CSMA/CA with high generation data rate

\subsubsection{Average Energy Consumption}

The state transition comprises of shutdown, ready, transmit and receive states [23] which consume minimum energy of BMSs. Due to the reduction in energy consumption, the CDASA-CSMA/CA uses this model in implementation as detailed in Table 1. Fig. 15 compares rounds-based energy consumption of BMSs of the CDASA-CSMA/CA with CSMA/CA. The LTA-MAC Superframe structure provides sufficient and dedicated channels to non-emergency and emergency-based BMSs. Also, the LTA-MAC provides sufficient time of $\mathrm{BI}$ to BMSs for contending and transmitting data which has reduced the repetition in rounds of the contention for accessing channel in the CDASA-CSMA/CA. However, these features are the limitations of the MAC Superframe structure of IEEE 802.15.4 and a CSMA/CA. At the beginning of the first round of contention, the CDASA-CSMA/CA needs $27 \mathrm{~mW}$ energy for transmission data which is the minimum as compared to a CSMA/CA. The achieved minimum energy consumption is $22 \%$ in the first round of the contention in CDASA-CSMA/CA, as shown in Fig. 15. The reason for this minimum energy consumption is the sufficient channels 
and dedicated slots for both types of a patient's data in the LTA-MAC Superframe structure. The repetition of rounds in the CSMA/CA increase the contention for accessing channels in CAP period. These increased numbers in rounds of contention consume more energy due to the limited channels. In this way, the performance of the MAC Superframe structure of IEEE 802.15.4 is degraded by causing a higher collision and BMSs retransmit the lost packets with a higher delay. Hence, the average energy consumption of BMSs in CDASA-CSMA/CA is reduced and saves energy up to $45 \%, 51 \%, 56 \%$ and $60 \%$ in the round 2 , round 3 , round 4 and round 5, respectively.

Fig. 16 compares the energy consumption of the body coordinators of the CDASA-CSMA/CA with a CSMA/CA. The LTA-MAC Superframe structure and CDASA-CSMA/CA scheme fulfill requirements of different types of a patient's data. The energy consumption of the body coordinator in the CDASA-CSMA/CA has been reduced and the body coordinator saves energy in the first round up to $40 \%$ as compared to a CSMA/CA. The energy consumption of the body coordinator in the remaining rounds is the minimum due to reduction in repetition of rounds of the contention for accessing channels as compared to CSMA/CA. Thus, the body coordinator saves energy in the second round is $90 \%$, the third round saves by $80 \%$, the fourth round saves by $70 \%$ and the fifth round saves by $68 \%$, as depicted in Fig 16 .

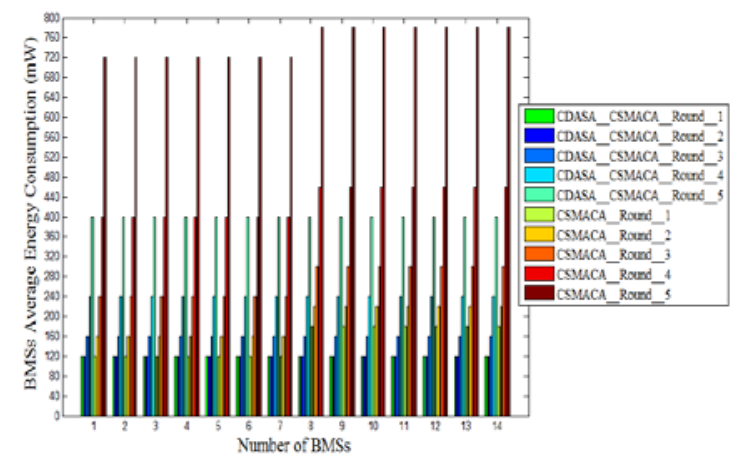

Fig. 15. Comparison of average energy consumption versus Number of BMSs between CDASA-CSMA/CA and CSMA/CA

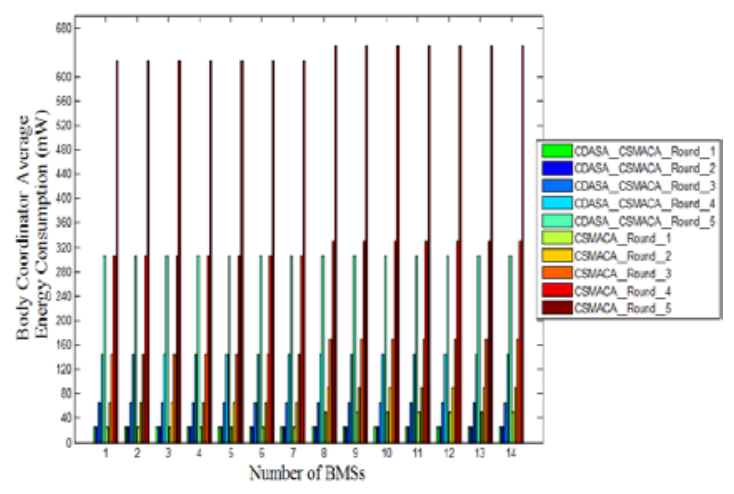

Fig. 16. Comparison of average energy consumption versus of the Body Coordinator between CDASA-CSMA/CA and CSMA/CA

\section{Conclusion}

The MAC Superframe structure of IEEE 802.15.4 provides 16 channels in the whole structure. The allocation of channels to BMSs is based on contention using a CSMA/CA. For accessing channel in CAP period using contention, the BMS repeats the values of each round of contention in the next rounds of contention. This degrades performance of the MAC Superframe structure of IEEE 802.15.4, causing higher collision and BMSs retransmit lost packets, which increases delay in the packet delivery, lower data reliability and consumes a maximum energy of BMSs. Our LTA-MAC Superframe structure protocol provides dedicated 128 slots for non-emergency an an emergency data. Also, in our CDASA-CSMA/CA scheme, we have used guardbands between channels to eliminate overlapping of the designated channels. Moreover, the BI of LTA-MAC allocates enough time to BMSs for contending to access CAP channel and transmission of data in the CFP's period; thereby, preventing BMSs 
from waiting for announcement of the next BI. The CDASA-CSMA/CA scheme reduces the repetition in rounds of contention using Equation (2). These reductions in rounds of contention increase the success rate of BMSs for accessing channel, which reduces collision of the packets by minimizing re-transmission of packets. These benefits reduce delay which consequently reduces energy consumption of BMSs.

\section{Conflict of Interests}

The authors declare that there is no conflict of interests regarding the publication of this paper.

\section{Acknowledgments}

The research is supported by Ministry of Higher Education Malaysia (MOHE) and conducted in collaboration with Research Management Center (RMC) at University Teknologi Malaysia (UTM) under VOT NUMBER: R.J130000.7828.4F859.

\section{References}

[1] L. Yu, L. Guo, H. Deng, K. Lin, L. Yu, W. Gao, and I. A. Saeed, "Research on Continuous Vital Signs Monitoring Based on WBAN," International Conference on Smart Homes and Health Telematics, pp. 371-382, 2016. Article (CrossRef Link)

[2] C. Chakraborty, B. Gupta, and S. K. Ghosh, "Tele-wound monitoring through smartphone," in Proc. of 2014 International Conference on Medical Imaging, m-Health and Emerging Communication Systems (MedCom), pp. 197-201, 2014. Article (CrossRef Link)

[3] M. M. Alam and E. Ben Hamida, "Surveying wearable human assistive technology for life and safety critical applications: Standards, challenges and opportunities," Sensors (Switzerland), vol. 14, pp. 9153-9209, 2014. Article (CrossRef Link)

[4] M. Shu, D. Yuan, C. Zhang, Y. Wang, and C. Chen, "A MAC Protocol for Medical Monitoring Applications of Wireless Body Area Networks,” Sensors, vol. 15, no. 6, pp. 12906-12931, 2015. Article (CrossRef Link)

[5] T. Maksymyuk, M. Kyryk, and M. Jo, "Comprehensive Spectrum Management for Heterogeneous Networks in LTE-U,” IEEE Wireless Communications, vol. 23, no. 6, pp. 1-8, Dec. 2016.

Article (CrossRef Link)

[6] S. Movassaghi, S. Member, M. Abolhasan, and S. Member, "Wireless Body Area Networks: A Survey," IEEE COMMUNICATIONS SURVEYS and TUTORIALS, vol. 16, no. 3, pp. 1658-1686, 2014. Article (CrossRef Link)

[7] M. Akbar, H. Yu, and S. Cang, "IEEE 802.15.4 Frame Aggregation Enhancement to Provide High Performance in Life-Critical Patient Monitoring Systems," Sensors, vol. 17, no. 2, pp. 1-25, Jan. 2017. Article (CrossRef Link)

[8] H. Su, S. Member, X. Zhang, and S. Member, "Battery-Dynamics Driven TDMA MAC Protocols for Wireless Body-Area Monitoring Networks in Healthcare Applications," IEEE Journal on Selected Areas in Communications, vol. 27, no. 4, pp. 424-434, 2009. Article (CrossRef Link)

[9] B. Liu, Z. Yan, and Chang Wen Chen, "CA-MAC: A Hybrid context-aware MAC protocol for wireless body area networks," in Proc. of 2011 IEEE 13th International Conference on e-Health Networking, Applications and Services, pp. 213-216, 2011. Article (CrossRef Link)

[10] P. Sthapit and J.-Y. Pyun, "Medium reservation based sensor MAC protocol for low latency and high energy efficiency,” Telecommunication Systems, vol. 52, no. 4, pp. 2387-2395, Apr. 2013. Article (CrossRef Link)

[11] M. Kim and C. H. Kang, "Priority-based service-differentiation scheme for IEEE 802.15.4 sensor networks in nonsaturation environments," IEEE Transactions on Vehicular Technology, vol. 59, 
no. 7, pp. 3524-3535, 2010. Article (CrossRef Link)

[12] R.-S. Cheng, C.-M. Huang, and T.-H. Tu, “A CSMA/CA-Based Media Access Protocol Using the Registered Backoff Time Vector (RBTv) Mechanism for M2M Communications,” Mobile Networks and Applications, vol. 22, no. 1, pp. 1-15, Jan. 2016. Article (CrossRef Link)

[13] S. Ullah and E. Tovar, "Performance analysis of IEEE 802.15.6 contention-based MAC protocol," in Proc. of 2015 IEEE International Conference on Communications (ICC), pp. 6146-6151, 2015. Article (CrossRef Link)

[14] S. Rashwand, J. Misic, and V. B. Misic, “Analysis of CSMA/CA Mechanism of IEEE 802.15.6 under Non-Saturation Regime,” IEEE Transactions on Parallel and Distributed Systems, vol. 27, no. 5, pp. 1279-1288, May 2016. Article (CrossRef Link)

[15] M. H. S. Gilani, I. Sarrafi, and M. Abbaspour, "An adaptive CSMA/TDMA hybrid MAC for energy and throughput improvement of wireless sensor networks,” Ad Hoc Networks, vol. 11, no. 4, pp. 1297-1304, Jun. 2013. Article (CrossRef Link)

[16] LAN/MAN Standards Committee, IEEE standard for Information Technology 802.15.4, Wireless Medium Access Control (MAC) and Physical layer (PHY) specifications for low-rate wireless personal area networks (LR-WPANs), 2006. Article (CrossRef Link)

[17] Z. a. Khan, M. B. Rasheed, N. Javaid, and B. Robertson, "Effect of Packet Inter-arrival Time on the Energy Consumption of Beacon Enabled MAC Protocol for Body Area Networks,” Procedia Computer Science, vol. 32, pp. 579-586, 2014. Article (CrossRef Link)

[18] J. S. Yoon, G.-S. Ahn, S.-S. Joo, and M. J. Lee, "PNP-MAC: Preemptive Slot Allocation and Non-Preemptive Transmission for Providing QoS in Body Area Networks,” in Proc. of 2010 7th IEEE Consumer Communications and Networking Conference, pp. 1-5, 2010. Article (CrossRef Link)

[19] I. Anjum, N. Alam, M. A. Razzaque, M. Mehedi Hassan, and A. Alamri, "Traffic Priority and Load Adaptive MAC Protocol for QoS Provisioning in Body Sensor Networks,” International Journal of Distributed Sensor Networks, vol. 2013, pp. 1-9, 2013. Article (CrossRef Link)

[20] M. Shakir, O. Rehman, M. Rahim, N. Alrajeh, Z. Khan, M. Khan, I. Niaz, and N. Javaid, "Performance Optimization of Priority Assisted CSMA/CA Mechanism of 802.15.6 under Saturation Regime,” Sensors, vol. 16, no. 9, p. 1421, Sep. 2016. Article (CrossRef Link)

[21] F. Xia, R. Hao, J. Li, N. Xiong, L. T. Yang, and Y. Zhang, “Adaptive GTS allocation in IEEE 802.15.4 for real-time wireless sensor networks,” Journal of Systems Architecture, vol. 59, no. 10, pp. 1231-1242, Nov. 2013. Article (CrossRef Link)

[22] S. Rezvani and S. A. Ghorashi, "Context aware and channel-based resource allocation for wireless body area networks,” IET Wireless Sensor Systems, vol. 3, no. 1, pp. 16-25, Mar. 2013. Article (CrossRef Link)

[23] J. Shuai, W. Zou, and Z. Zhou, "Priority-based adaptive timeslot allocation scheme for wireless body area network," in Proc. of 2013 13th International Symposium on Communications and Information Technologies (ISCIT), pp. 609-614, 2013. Article (CrossRef Link)

[24] J. Zhou, A. Guo, J. Xu, and S. Su, “An optimal fuzzy control medium access in wireless body area networks,” Neurocomputing, vol. 142, pp. 107-114, Oct. 2014. Article (CrossRef Link)

[25] D. Naranjo-Hernández, L. M. Roa, J. Reina-Tosina, M. A. Estudillo-Valderrama, and G. Barbarov, "Low-Power Platform and Communications for the Development of Wireless Body Sensor Networks,” International Journal of Distributed Sensor Networks, vol. 2015, pp. 1-13, 2015. Article (CrossRef Link)

[26] T. Issariyakul and E. Hossain, Introduction to Network Simulator NS2, Second. Boston, MA: Springer US, 2012. Article (CrossRef Link) 


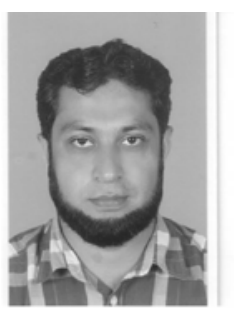

Fasee Ullah received the MS degree in computer science from SZABIST, Pakistan, in 2009. In 2017, Fasee ullah received $\mathrm{PhD}$ degree in computer science from Universiti Teknologi Malaysia (UTM), Johor Bahru, Malaysia. He has 6 years teaching experience. Further, he has 35 research publications in various reputed journals and conferences. His research interests include: Wireless Body Area Networks, WSNs, Cloud Computing, and Internet of Things (IoTs).

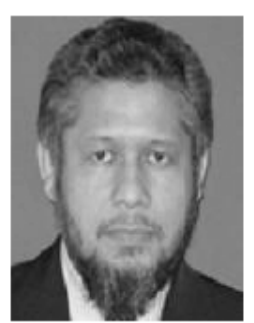

Abdul Hanan Abdullah received the Ph.D. degree from Aston University, Birmingham, U.K., in 1995. From 2004 to 2011, he was the Dean of the Faculty of Computer Science and Information Systems. He is currently heading the Pervasive Computing Research Group under K-Economy Research Alliances, Universiti Teknologi Malaysia, Johor Bahru, Malaysia, where he is a Professor. His research interests include wireless sensor networks, mobile ad hoc networks, network security, and next-generation networks.

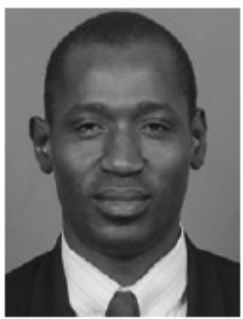

Gaddafi Abdul-Salaam received the Ph.D. degree from the Universiti Teknologi Malaysia, Johor, Malaysia in 2017. He received his M.Sc. degree in Advanced ICT studies from the Institute for Advanced ICT Studies, Ghana in 2009, and B.Sc. degree in Computer Engineering from the Kwame Nkrumah University of Science and Technology (KNUST), Ghana in 2005. He was also head of the ICT Centre in KNUST from 2010-2013. He is now a lecture at the Department of Computer Science, KNUST. His research interests include Wireless Sensor Networks (WSN), Mobile Ad hoc Networks and Cloud Computing.

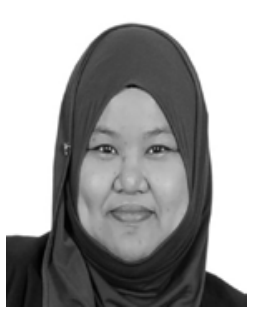

Marina Md Arshad received her M.Sc. degree in Data Communications, Network and Distributed Systems from University College London, UK and B.Sc in Computer Science from Universiti Teknologi Malaysia. She is a member of the Pervasive Computing Research Group in Universiti Teknologi Malaysia. Her current research interests are Optical Burst Switching Network and Network Performance Analysis.

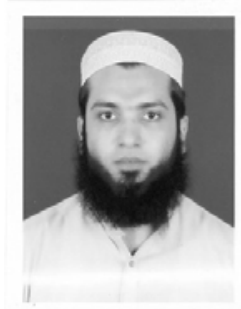

Farhan Masud received MS in Computer Science degree from the Computer Science Department, Governement College University, Lahore, Pakistan, in 2014. He is currently working towards the $\mathrm{PhD}$ degree in wireless body area networks in the Faculty of Computing, Universiti Teknologi Malaysia, Johor Bahru, Malaysia. Farhan Masud is a Senior Assitant Professor at the Department of Statistics and Computer Science, Faculty of Life Sciences Business Management, University of Veterinary \& Animal Sciences, Lahore, Pakistan. He has been the Head of the Department at the Department of Statistics and Computer Science, Faculty of Life Sciences Business Management, University of Veterinary \& Animal Sciences, Lahore, Pakistan for eight year from 2006 to 2014. 